\title{
Análisis de las demandas de Sprint en competición por puestos específicos en el rugby 7 femenino Analysis of the Sprint demands during competition by playing positions in women's rugby 7 \\ Javier Rodríguez-Baena, Javier Gálvez-González \\ Universidad Pablo de Olavide (España)
}

\begin{abstract}
Resumen. El objetivo de este estudio es analizar las manifestaciones de sprint, la capacidad de repetir sprint y las posibles diferencias entre los periodos de juego de jugadoras de rugby 7, teniendo en cuenta su puesto específico de juego. Fueron monitorizadas 21 jugadoras (edad 21.2 \pm 2.4 ) agrupadas por puestos específicos: delanteras $(\mathrm{n}=9 ; 166.3 \pm 12.6 \mathrm{~cm} ; 65.4 \pm 7.2 \mathrm{~kg})$ y línea $3 / 4(\mathrm{n}=12 ; 163.4 \pm 9.8 \mathrm{~cm}$; $61.7 \pm 4.1 \mathrm{~kg}$ ) en torneos de competición oficial, utilizando un sistema de posicionamiento global (GPS). Para determinar un sprint se consideró umbral individualizado del $61 \%$ de la velocidad máxima de cada jugadora. Los resultados muestran que existen diferencias significativas al analizar los grupos de jugadoras $(p<.05)$, en la velocidad máxima $(27.0 \pm 2.10$ vs $24.7 \pm 1.65)$, en velocidad máxima en competición $(25.9 \pm 2.34$ vs $21.9 \pm 2.59)$, umbral de sprint $(16.4 \pm 1.29$ vs $15.0 \pm 1.0)$, número de sprint $(6.83 \pm 1.88$ vs $4.54 \pm 1.44)$, distancia recorrida a sprint $(166.0 \pm 15.64$ vs $135 \pm 13.94)$ y sprint repetidos $(1.82 \pm .76$ vs $1.11 \pm .31)$. Se encontraron diferencias significativas entre ambos periodos de juego $(p<.05)$, observándose una disminución considerable en el segundo tiempo de la velocidad máxima en competición, el número de sprints, distancia recorrida a sprint y sprints repetidos. Podemos concluir que el uso de umbrales relativos incrementa el registro del número de sprint. Las diferencias por puestos específicos puede ayudarnos a una mejor comprensión de las demandas de competición y optimizar la planificación del entrenamiento.
\end{abstract}

Palabras clave. Velocidad máxima, puestos específicos, competición, GPS.

\begin{abstract}
The aim of this study is to analyze sprint demands, the ability to repeat sprints and the possible differences between the playing periods of rugby-7 players, considering their playing position. A total of 21 players (age $21.2 \pm 2.4$ ) grouped by specific positions (forwards: $\mathrm{n}=9 ; 166.3 \pm 12.6 \mathrm{~cm} ; 65.4 \pm 7.2 \mathrm{~kg}$; and backs $: \mathrm{n}=12 ; 163.4 \pm 9.8 \mathrm{~cm} ; 61.7 \pm 4.1 \mathrm{~kg}$ ) were monitored in official competition tournaments, using a global positioning system (GPS). To determine a sprint, an individual threshold of $61 \%$ of the maximum speed of each player was considered. The results show that, when analyzing the group of players $(p<.05)$, there are significant differences in the maximum speed ( $27.0 \pm 2.10$ vs $24.7 \pm 1.65)$, in maximum speed in competition ( $25.9 \pm 2.34$ vs $21.9 \pm 2.59)$, threshold of sprint $(16.4 \pm 1.29$ vs $15.0 \pm 1.0)$, sprint number $(6.83 \pm 1.88$ vs $4.54 \pm 1.44)$, distance traveled to sprint $(166.0 \pm 15.64 \mathrm{vs} 135 \pm$ $13.94)$, and repeated sprint $(1.82 \pm .76$ vs. $1.11 \pm .31)$. Significant differences were found between both periods of play $(p<.05)$, observing a considerable decrease of the maximum speed in competition, the number of sprints, distance traveled to sprints, and repeated sprints during the second half. The use of relative thresholds increases the number of sprints. The differences in playing positions can help us to better understand the demands of competition and optimize training planning.
\end{abstract}

Key words. Maximum speed, playing positions, competition, GPS.

\section{Introducción}

La velocidad en el juego es una de las principales características del rugby 7 , la modalidad olímpica de este deporte. Las condiciones de la competición, con siete jugadores en el mismo espacio de juego que en rugby 15 , demanda a los jugadores una intensidad muy alta en sus acciones y la repetición de esfuerzos a alta intensidad, alternados con cortos periodos de recuperación (Meir, 2012). La carrera de alta velocidad es transcendental en el rugby 7 (Blair et al., 2017), tanto en fases defensivas (Barkell et al., 2017) como en fases de ataque (Wheeler \& Sayers, 2011); así como también en el momento de la evasión de un placaje (den Hollander et al., 2016) y la consecución de un ensayo (Smart et al., 2014). En fútbol masculino, (Al Haddad et al., 2015) se ha estimado la diferencia entre la velocidad máxima de los jugadores y la velocidad máxima alcanzada en competición, a modo de indicador de si los valores máximos de esta capacidad pueden tener un efecto sobre el rendimiento en el juego, o si la dinámica del mismo permite al jugador expresar sus valores máximos de carrera. Sin embargo, no hay estudios similares realizados en rugby 7 femenino, y por tanto los entrenadores carecen de valores de referencia sobre la importancia de la velocidad en el juego.

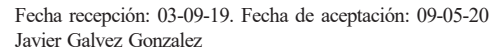

Es importante que entrenadores y preparadores físicos conozcan las demandas de los jugadores en competición y entrenamiento (Scott et al., 2016) para poder programar correctamente la preparación de los deportistas. En jugadoras de élite de rugby 7 la distancia media recorrida es de 1468 metros (Clarke et al., 2017), de los cuales la distancia a alta intensidad puede alcanzar los $199 \mathrm{~m}$ (Malone et al., 2018), pudiendo llegar el porcentaje de distancia recorrida a alta intensidad hasta el $15-25 \%$ del total (Vescovi \& Goodale, 2015). Durante la competición, el número de sprints realizados pueden llegar a ser $\sim 5-6$, con una velocidad máxima que puede oscilar entre los 23-24,9 km/ $\mathrm{h}^{-1}$ - (Portillo et al., 2014; Suarez-Arrones, Nuñez, Portillo, \& Mendez-Villanueva, 2012), con una distancia media recorrida a sprint de $148 \mathrm{~m}$ durante un partido (Clarke et al., 2017). Durante el juego, los jugadores pueden necesitar repetir estas acciones en cortos periodos de tiempo, por lo que la capacidad de repetición de sprints puede ser un elemento fundamental en este deporte (Higham et al., 2016), produciéndose en torno a 3 por partido (SuarezArrones et al., 2016). Si bien su valor total no es elevado, si lo es al relativizarlo sobre el número total $(\mathrm{n}=8)$, pues supone un $37,5 \%$, lo que indica que cuando los jugadores son requeridos para hacer un sprint, en breve tiempo son requeridos para realizar otro sin haberse recuperado del anterior

En el rugby 7, al igual que en otros deportes femeninos (Leal-Cussaria et al., 2019), se puede apreciar que a lo largo del partido puede aparecer una disminución en el rendimiento de carrera si comparamos los parámetros de primera parte 
y segunda parte (Portillo et al., 2014), lo cual indica una posible aparición de fatiga, reflejo de la intensidad de los esfuerzos realizados.

Los estudios antes citados, indican que las jugadoras realizan un número de sprints que puedes ser considerado bajo, y sin embargo, la observación directa del juego parece contradecirlo. Esto puede ser debido a que la mayoría de los estudios relacionados con el rugby 7 han sido realizados con jugadores masculinos, y se han usado los mismos umbrales de velocidad para analizar la velocidad en las jugadoras. El uso de estos umbrales puede implicar una estimación incorrecta de las demandas de carrera a altas velocidades de las jugadoras, debido a que la metodología utilizada para ello suele estar diseñada para deportistas masculinos (Hodun et al., 2016). En el caso del fútbol, diferentes estudios avalan el uso de umbrales individuales en el análisis por gps tanto de la velocidad (Núñez-Sánchez et al., 2017) como de las aceleraciones (Núñez et al., 2018). Por tanto, para llegar a interpretar más adecuadamente los diferentes parámetros de rendimiento, se empieza a sugerir que deberíamos tener en cuenta los valores relativos a las capacidades individuales de cada jugadora para de esta forma eliminar posibles subestimaciones relacionada con la velocidad de cada jugador (Murray et al., 2018).

En ésta línea de trabajo, Gabbett (2015) compara el uso de un umbral absoluto de $5 \mathrm{~m} \cdot \mathrm{s}^{-1}$ frente al uso de un umbral relativos del $70 \%$ de la velocidad máxima, y entonces, los jugadores de rugby sub 15 aumentan de 2,7 a 6,8 sus actividades de alta intensidad; y (Reardon et al., 2015) cuando comparan el valor absoluto de $5 \mathrm{~m} \cdot \mathrm{s}^{-1}$ frente al relativo del $60 \%$ de la velocidad máxima, los delanteros (más pesados y altos) incrementan la distancia recorrida a alta intensidad de 269 a 354 m, mientras que los jugadores de la línea de 3/4, descienden sus valores de 697 a $570 \mathrm{~m}$. Podemos entender que si se usan valores absolutos, los jugadores más lentos obtienen valores medidos que en realidad subestiman los esfuerzos realizados durante el partido, pues se estarán anotando resultados obtenidos con la misma intensidad de carrera a deportistas cuya velocidad máxima es distinta, de forma que los más rápidos tendrán que utilizar un porcentaje relativamente bajo de su velocidad máxima para anotar la realización de un sprint, mientras que los deportistas más lentos realizarán esfuerzos relativamente altos para ser considerados igual. Por ejemplo, si se usa un valor absoluto de $20 \mathrm{~km} \cdot \mathrm{h}^{-1}$ para anotar un sprint, un jugador de rugby $7 \mathrm{con}$ una velocidad máxima de $35 \mathrm{~km} \cdot \mathrm{h}^{-1}$ (Jiménez-Reyes et al., 2019) verá anotado un sprint cuando supera el $57 \%$ de su velocidad. Pero si una jugadora (Ortega-Gálvez et al., 2019) tienen una velocidad máxima de 27 , para anotar un sprint deberá superar el 74\% de su VMax.

Diferentes estudios han usado el valor absoluto o umbral de $20 \mathrm{~km} \cdot \mathrm{h}^{-1}$ (Suarez-Arrones et al., 2012; Suarez-Arrones, Portillo, et al., 2014), sin embargo, (Buchheit, MendezVillanueva, Simpson, \& Bourdon, 2010) proponen establecer como umbral relativo del sprint alcanzar el $61 \%$ de la velocidad máxima del deportista. A esta velocidad, se puede observar en jugadores de rugby de alto nivel un patrón de carrera con fase aérea y con un esfuerzo de brazos intenso (Duthie et al., 2005).

En el rugby, la existencia de algunas acciones del juego, como la melé, determina la existencia de unos puestos específicos muy determinados, y que exigen muchas veces características antropométricas especiales. Aunque en el rugby 7 hay menos diferencias entre los perfiles físicos y antropométricos, existen controversias sobre la necesidad de examinar de forma diferenciada las demandas de los 3/4 y delanteros. Algunos autores indican que las delanteras son más altas y pesadas (170.5 cm vs $166.3 ; 72.87 \mathrm{~kg}$ vs 66.4 respectivamente), mientras que las jugadoras de la $3 / 4$ son más rápidas $\left(29.55 \mathrm{~km} \cdot \mathrm{h}^{-1} \mathrm{vs} 28,72 \mathrm{~km} \cdot \mathrm{h}^{-1}\right)$; sugiriendo además el empleo de programas de entrenamientos similares para ambos grupos (Agar-Newman et al., 2017). Estas diferencias antropométricas podrían explicar las diferencias en las demandas físicas y fisiológicas encontradas tanto en jugadores masculinos (Suarez-Arrones, Arenas, et al., 2014) como femeninos (Malone et al., 2018). Éstos indican que en jugadoras élite, las jugadoras de la 3/4 presentan mayores valores en relación con las distancias recorridas a alta intensidad de carrera y sprints, el número de sprints y la máxima distancia recorrida a sprint.

Una vez revisada la literatura científica, se observa que no existe información precisa sobre la velocidad máxima de las jugadoras de rugby 7 en competición, adaptada a las capacidades de cada jugadora, a su puesto específico y a lo largo del partido. Por ello, nuestro objetivo será analizar las demandas de competición de forma individualizada en el rugby 7 femenino.

\section{Método}

\section{Sujetos}

Participaron en el estudio 21 jugadoras (edad 21.2 \pm 2.4 ) de rugby 7 femenino pertenecientes al mismo club, el cual sólo compite en esta modalidad de rugby, en el cual está especializado, jugando en la máxima categoría nacional española. Para el análisis del juego, las jugadoras fueron agrupadas por los dos grupos de puestos específicos existentes en Rugby 7 : delanteras ( $\mathrm{n}=9 ; 166.3 \pm 12.6 \mathrm{~cm} ; 65.4 \pm 7.2 \mathrm{~kg})$ y línea de $3 / 4(\mathrm{n}=12 ; 163.4 \pm 9.8 \mathrm{~cm} ; 61.7 \pm 4.1 \mathrm{~kg})$. Todas las jugadoras entrenan 4 días por semana incluyendo entrenamientos de campo y de gimnasio. Las jugadoras fueron informadas del objetivo final del estudio, y aceptaron participar en el mismo, firmando el consentimiento informado.

\section{Instrumentos y Procedimiento}

Se llevó a cabo un estudio cuasiexperimental, descriptivo, con una selección incidental de la muestra, en el que no se manipulan de las variables. Los datos de movimiento de los partidos se obtuvieron mediante el uso de dispositivos GPS Spi HPU (GPS Sport System, Camberra, Australia). El GPS se coloca en un arnés de neopreno en la espalda de las jugadoras, justo entre las dos escápulas. Conforme a las especificaciones del fabricante, el sensor GPS tiene una velocidad de muestreo de $15 \mathrm{~Hz}$. Los datos registrados por el dispositivo fueron exportados al programa Team AMS (versión R1 2019.1) provisto por el fabricante. Este dispositivo ha sido validado para establecer las demandas de carrera en deportes de equipo (Scott et al., 2016). Las jugadoras llevaron siempre el mismo GPS a lo largo de los diferentes partidos para minimizar el error de medida (Jennings et al., 2010). 
El GPS se encendió 30 minutos antes del inicio de cada partido, los cuales fueron jugados en estadios abiertos para asegurar la conectividad a los satélites.

Se obtuvieron datos de 21 torneos de competición oficial a lo largo de las temporadas 2015-16, 2016-17 y 2017-18. Sólo se analizaron los datos de las jugadoras que completaron todo el partido, siendo por tanto la muestra de 82 archivos, existiendo por tanto datos de diferentes jugadoras en diferentes momentos. Varias jugadoras formaron parte de la plantilla del equipo durante las tres temporadas.

Para el análisis de los datos de carrera de las jugadoras se tomaron los siguientes valores de referencia. La velocidad máxima de sprint (VMS) de cada jugadora fue obtenida de los registros del GPS a lo largo de los tests de velocidad medidos en los entrenamientos, realizados la semana previa a cada torneo. Las mediciones se llevaron a cabo a la misma hora, en la misma instalación y asegurando las mismas condiciones de viento. Para ello las jugadoras realizaban dos sprints de 50 metros (anotando el mejor de los tiempos), asegurando un descanso de 5 minutos entre cada repetición. Se decidió esta distancia, pues es la única que asegura que todos los jugadores seniors han alcanzado su velocidad máxima (Zabaloy et al., 2020), pues $\sim 50 \%$ de los jugadores la alcanza antes de los $30 \mathrm{~m}, \sim 35 \%$ a los $40 \mathrm{~m}$ y $\sim 15 \%$ a los $50 \mathrm{~m}$. El uso del GPS para medir el sprint en jugadores de rugby 7 ha sido previamente validado (Lacome et al., 2019). Al analizar los partidos, se anotó un sprint cada vez que las jugadoras alcanzaban el $61 \%$ de su VMS y era mantenido al menos durante $1 \mathrm{~s}$. Este valor umbral ha sido previamente usado por (Buchheit et al., 2010) como umbral individualizado, ya que a esa velocidad es cuando podemos considerar un sprint realizado por jugadores de rugby de alto nivel (Duthie et al., 2006), cuando el jugador parte de una posición estática erguida. Se calculó la ratio entre la VMS de la jugadora con la VMS alcanzada durante los partidos (VMScomp) para estimar las demandas de la competición.

Para establecer la capacidad de repetir las acciones de sprint, se anotaba un sprint repetido cada vez que se realizaban dos sprints consecutivos en un intervalo de tiempo de 30 s (Suarez-Arrones et al., 2016).

\section{Análisis estadístico}

Para determinar la distribución de la muestra se empleó el test de Shapiro-Wilk, el cual indicó que todas las variables provienen de una población Normal $(p>.05)$. Para comprobar que las variables de rendimiento provienen de una población normal, se realizo la prueba de Kolmogorov-Smirnoff $(p>.05)$. Para establecer las posibles diferencias entre los dos grupos de jugadoras se usó la prueba t para medidas independientes, con un nivel de significación $p<.05$. Se aseguraron las condiciones de independencia, aleatoriedad (test de las rachas) y homocedasticidad (test de Levene). Las diferencias en las variables de carrera entre la primera y la segunda parte se analizaron con la prueba t para medidas apareadas, con un nivel de significación de $p<.05$. Se aseguraron la condición de aleatoriedad (test de las rachas). La magnitud de las diferencias se determinó mediante el tamaño del efecto (TE), el cual fue calculado usando los valores expuestos por Cohen (1988) como trivial (0.0-0.19), pequeño (0.2-0.59), moderado(0.6-1.1), largo(1.2-1.9) y muy largo (>2.0).

\section{Resultados}

El grupo de las delanteras tiene una mayor altura (166.3 cm vs $163.4 \mathrm{~cm}, p<.05)$ y un mayor peso $(65.4 \mathrm{~kg}$ vs $61.7 \mathrm{~kg}$, $p<.05)$ lo que refleja unos requerimientos antropométricos diferentes para los dos grupos de jugadoras.

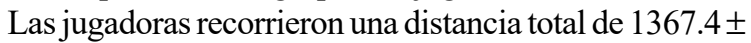
$124.2 \mathrm{~m}$ a lo largo de los partidos, repartidos existiendo diferencias entre la primera parte y la segunda $(715.4 \pm 68.1 \mathrm{vs}$ $620.0 \pm 52.6 ; p<.001)$.

La media de las VMS de las jugadoras fue de $26.0 \pm 2.29$ $\mathrm{km} \cdot \mathrm{h}^{-1}$, y la media de las velocidades máximas alcanzadas durante la competición fue $24.2 \pm 3.24 \mathrm{~km} \cdot \mathrm{h}^{-1}$, lo cual supone que las jugadoras alcanzan el $92.87 \%$ de su VMS cuando compiten. El umbral para anotar un sprint (61\% de VMS) tuvo un valor medio de $15.8 \pm 1.39 \mathrm{~km} \cdot \mathrm{h}^{-1}$, y se realizaron de media $5.85 \pm 2.1$ sprints por partido y jugadora, así como 1,57 $\pm 0,75$ sprints repetidos.

La distancia recorrida durante los sprints fue de $152.76 \pm$ 21.87 , lo cual supone un $11.17 \%$ de la distancia total.

Tabla 1

Diferencias (media \pm desviación estándar) en manifestaciones de Velocidad entre las delanteras y la línea de $3 / 4$

\begin{tabular}{lcccc}
\hline & Delanteras n=10 & Línea $3 / 4 \mathrm{n}=9$ & $P$ valor & $\begin{array}{c}\text { Tamaño Efecto } \\
(90 \% \text { LC })\end{array}$ \\
\hline VMS & $24.7 \pm 1.65$ & $27.0 \pm 2.10$ & .015 & $1.12(.40-1.84)$ \\
VMScomp & $21.9 \pm 2.59$ & $25.9 \pm 2.34$ & .003 & $1.48(.73-2.23)$ \\
Umbral Sprint (61\% VMS) & $15.0 \pm 1.0$ & $16.4 \pm 1.29$ & .016 & $1.1(.38-1.83)$ \\
$\mathrm{N}^{\circ}$ Sprint & $4.54 \pm 1.44$ & $6.83 \pm 1.88$ & .007 & $2,3(1-3.6)$ \\
Distancia recorrida a sprint & $135 \pm 13.94$ & $166.0 \pm 15.64$ & .000 & $1.92(1.19-2.64)$ \\
Sprint Repetidos & $1.11 \pm .31$ & $1.82 \pm .76$ & .006 & $1.26(.56-195)$ \\
\hline VMS: Velocidad Máxima Sprint; VMScomp: Velocidad Máxima en competición; LC: Limites \\
de Confianza
\end{tabular}

La tabla 1 muestra las diferencias encontradas en las manifestaciones de sprint en las jugadoras diferenciadas por puestos específicos. Las delanteras sólo alcanzan en competición el $88.57 \%$ de su VMS, mientras que las jugadoras de la línea de $3 / 4$ casi llegan a lograr los mismos valores de su VMS, pues alcanzan el $96.09 \%$.

\begin{tabular}{|c|c|c|c|c|}
\hline & Primera Parte & Segunda Parte & $P$ valor & $\begin{array}{c}\text { Tamaño Efecto } \\
(90 \% \text { LC) }\end{array}$ \\
\hline VMScomp & $23.6 \pm 3.1$ & $23.8 \pm 3.41$ & .43 & $-.24(-.75-.27)$ \\
\hline $\mathrm{N}^{\circ}$ Sprint & $3.43 \pm 1.28$ & $2.42 \pm 1.31$ & .015 & $-.76(-1.77--.25)$ \\
\hline Distancia recorrida a sprint & $81.5 \pm 13.22$ & $71.7 \pm 10.81$ & .014 & $-.77(-1.28--.26)$ \\
\hline Sprint Repetidos & $1.24 \pm .34$ & $.33 \pm .28$ & .000 & $-1.93(-2.44--1.42)$ \\
\hline
\end{tabular}

\section{Discusión}

El objetivo de este estudio era analizar las manifestaciones de sprint de jugadoras de rugby 7 , teniendo en cuenta su puesto específico, usando como umbral individualizado el $61 \%$ de la VMS de cada jugadora, así como la capacidad de repetir sprint. Igualmente, se analizó las posibles diferencias entre los tiempos de juego.

Las jugadoras muestran una adecuada condición física específica para este deporte, pues alcanzan a recorrer 1367.4 $\mathrm{m}$ a lo largo de un partido, valor similar (1468 m) al alcanzado por jugadoras de élite internacional (Clarke et al., 2017). Teniendo en cuenta el uso de un umbral relativo a la hora de determinar un sprint, la distancia recorrida a sprint es del $11.17 \%$ de la distancia total, lo cual indica asimismo una correcta adaptación al metabolismo no oxidativo requeridos para realizar esfuerzos de alta intensidad. Estos resultados muestran una notable diferencia sobre otros trabajos en rugby 7 femenino de élite (Portillo et al., 2014; Suarez-Arrones et al., 2012) que utilizan un valor absoluto de sprint $\left(20 \mathrm{~km} / \mathrm{h}^{-1}\right)$, 
recorriendo un $\sim 45 \%$ menos de distancia a sprint que nuestras jugadoras. El uso de este valor absoluto de umbral conlleva una inadecuada estimación de los registros relacionados con la distancia total de sprint, provocando una subestimación de los esfuerzos desarrollados en competición por las jugadoras. Estas diferencias reflejan la importancia del uso de valores relativos frente a valores absolutos para conseguir una adecuada evaluación de los parámetros relacionados con la velocidad durante un partido (Ortega-Gálvez et al., 2019).

Cuando se analiza la VMS, los resultados muestran que las jugadoras de la línea de $3 / 4$ son más rápidas que las delanteras $\left(27.0 \mathrm{~km} / \mathrm{h}^{-1}\right.$ frente a $\left.24.7 \mathrm{~km} / \mathrm{h}^{-1}\right)$, lo que supone un pico de velocidad $\sim 8 \%$ mayor. Estudios que analizan las demandas competitivas en ambos grupos posicionales (Goodale et al., 2016; Malone et al., 2018) también revelan porcentajes diferenciadores de la velocidad entre ambos grupos, donde las delanteras son más lentas $(\sim 2-6 \%)$ que las $3 / 4$. Existen evidencias para afirmar que la disposición de las jugadoras en el terreno de juego condiciona su velocidad máxima en competición. De modo que, para mejorar nuestra comprensión de la VMS deberíamos tener presente una evaluación individualizada de las demandas competitivas por puestos específicos.

En el análisis de cualquier demanda por puestos específicos, no debemos obviar las características antropométricas de las jugadoras (Agar-Newman et al., 2017). Los datos recogidos en este estudio muestran una disparidad antropométrica, donde las delanteras son más altas $(\sim 2 \%)$ y más pesadas $(\sim 6 \%)$ que las jugadoras de la línea de $3 / 4$. AgarNewman et al., (2017) también reflejan un mayor peso y altura en el grupo de las delanteras. Aunque, la mayoría de las jugadoras de rugby 7 tienen unos parámetros antropométricos similares (Clarke et al., 2017; Portillo et al., 2014; Suarez-Arrones et al., 2012); parece necesario continuar distinguiendo a las jugadoras en relación a sus características antropométricas (Ross et al., 2014). Este análisis diferenciado por puestos específicos ayudará a entrenadores y preparadores físicos a entender con una mejor precisión las características individualizadas de las jugadoras y mejorar la planificación del entrenamiento de cada grupo posicional.

Las jugadoras en competición no llegan a alcanzar su velocidad máxima mostrada en los test, pero consiguen acercarse hasta el $92.87 \%$ de su VMS. Análogamente ocurre en otros trabajos, donde las jugadoras llegan a alcanzar el 94,61\% (en nivel nacional) y el $97.06 \%$ (en nivel internacional) de su velocidad máxima en competición (Vescovi \& Goodale, 2015). Aunque una jugadora en un partido puede tener la ventaja en el momento de iniciar su carrera de empezar desde una situación no estática, las acciones de compañeras y contrarias pueden impedir que una jugadora llegue a alcanzar el pico máximo de su capacidad. De tal forma que, las jugadoras de la línea de 3/4 consiguen aproximarse bastante a su VMS en comparación con las delanteras ( $96.09 \%$ frente a $88.57 \%)$. Una mayor VMS de las $3 / 4\left(27.0 \mathrm{~km} \cdot \mathrm{h}^{-1}\right.$ frente a 24,7$)$ puede hacer que una jugadora sea capaz de superar a la defensa, y al escaparse, tener suficiente espacio para poder expresar su máxima capacidad de velocidad. En esta misma línea, Smart, Hopkins, Quarrie \& Gill (2014) exponen que los jugadores más rápidos rompen la línea de defensa, evadiendo a sus defensores y marcando ensayos con más frecuencia que los delanteros. Las delanteras participantes en éste estudio son más altas y pesadas $(166.3 \mathrm{~cm}$ vs $163.4 \mathrm{~cm} ; 65.4 \mathrm{~kg}$ vs 61.7 $\mathrm{kg})$, y menos rápidas $\left(24.7 \pm 1.65 \mathrm{~km} \cdot \mathrm{h}^{-1}\right.$ vs $27.0 \pm 2.10 \mathrm{~km} \cdot \mathrm{h}^{-}$ $\left.{ }^{1}\right)$, y por ello su aportación al juego es diferente. Ross et al., (2015) muestran como los delanteros en rugby 7 alcanzan menos velocidad en el juego, realizan más placajes, intervienen en más rucks defensivos, además de intervenir en las melés y los saques de lateral, y anotan menos ensayos. Además, rompen menos la línea de placaje, por lo que tienen menos posibilidades de correr distancias más largas en las que poder alcanzar la Vmax.

Por ello, el conocimiento de la velocidad máxima específica requerida para cada grupo posicional es un parámetro de rendimiento clave en este deporte, pudiendo incluso determinar el resultado final de un partido (Portillo et al., 2014).

La capacidad de un deportista de ejecutar un sprint es esencial para el rendimiento en los deportes de equipo (Sweeting et al., 2017) y con especial relevancia en el rugby 7 (Schuster et al., 2018). En este estudio, las jugadoras realizaron un total de 5.85 sprints de media por partido, que si las diferenciamos por puestos específicos, las delanteras realizan 4.54 sprints y la línea de $3 / 4$ ejecutan 6.83 sprints. Coincidiendo con otros trabajos en rugby 7 masculino (Higham et al., 2016) y rugby 7 femenino (Malone et al., 2018), donde el número de sprint es mayor en el grupo de las jugadoras de $3 / 4$. Estas diferencias pueden responder a factores técnicos, tácticos y físicos, ya que las delanteras se involucran con una menor frecuencia en acciones de juego que implican pases de balón y alta velocidad (Ross et al., 2015).

Las jugadoras estudiadas realizan un mayor número de sprints que en otros estudios, tanto en jugadoras de su mismo nivel competitivo (Portillo et al., 2014), como en otras que compiten en nivel internacional (Suarez-Arrones et al., 2012). De la misma forma, ocurre en el único estudio por puestos específicos en jugadoras de nivel internacional (Malone et al., 2018), donde el porcentaje de sprints realizados es menor en las $3 / 4(-34,11 \%)$ y en las delanteras $(-44.93 \%)$. Una de las causas que puede explicar estas diferencias, está relacionado con el uso de umbrales de velocidad adecuados. En el presente trabajo, para determinar un sprint se fijó un umbral relativo del $61 \%$ de la velocidad máxima alcanzada por las jugadoras (Buchheit, Mendez-villanueva, Simpson, y Bourdon, 2010), estableciéndose el umbral en el presente estudio en $15.8 \mathrm{~km} / \mathrm{h}$. Este umbral relativo supone el $79 \% \mathrm{del}$ valor absoluto $\left(20 \mathrm{~km} / \mathrm{h}^{-1}\right)$, utilizado por la gran mayoría de literatura científica. Con la utilización de este umbral absoluto, no se habrían anotado el $21 \%$ de sprints desarrollados por las jugadoras. Estas diferencias en la estimación de un sprint con un umbral absoluto de velocidad, puede suponer la subestimación de valores de alta intensidad en las jugadoras más lentas y la sobreestimación en las más rápidas (Gabbett, 2015; Reardon et al., 2015). De esta manera, el uso de un umbral relativo, pueda ser una opción que muestre un mayor ajuste a la realidad de los sprints realizados por las jugadoras durante un partido.

Este trabajo es el primero que analiza el número de sprint repetidos por puestos específicos en rugby 7 femenino, reflejando datos contrarios a los recogidos en un trabajo de rugby unión masculino, donde el número de sprints repeti- 
dos en los jugadores es mayor y los delanteros realizan más que los $3 / 4$ (Austin et al., 2011) . Aunque éste último estudio, utiliza el video como método de registro de datos, y tiene en cuenta otras variables como son patrones de contacto propios del rugby, mientras que el presente estudio se ha utilizado tecnología GPS para evaluar las demandas competitivas. Estas diferencias pueden responder a la implicación de cada grupo en esfuerzos que generen un alto coste fisiológico, como son las acciones de contacto (placajes, scrums y rucks), sprints y esfuerzos de alta intensidad (Austin et al., 2011).

La pérdida de rendimiento entre ambos periodos durante los diferentes torneos, indica un claro descenso en el segundo tiempo de las diferentes variables analizadas como el número de sprint realizados (-29.4\%), en la distancia recorrida a sprint (-12.02\%) y en los sprint repetidos (-73.3\%). Diversos estudios (Goodale et al., 2017; Malone et al., 2018; Portillo et al., 2014) indican que la distancia recorrida a sprint disminuye entre la primera parte y la segunda, al igual que ocurre en el rugby 7 masculino con un descenso de los sprint repetidos (Suarez-Arrones et al., 2016). Estos datos muestran acumulación de fatiga y acompañada de falta de capacidad para mantener esfuerzos de alta intensidad; un factor determinante en el rugby 7 .

\section{Conclusiones}

Con los datos recogidos en el presente estudio, se puede afirmar que el uso de un umbral relativo de velocidad aporta una información más ajustada a la realidad, en relación con las manifestaciones de sprint en el rugby 7 femenino. El estudio aporta como referencia más relevante el registro de un mayor número de sprints durante un partido, existiendo una notable diferencia con otros estudios que han analizado el rugby 7 femenino.

Además, una adecuada compresión por puestos específicos de la velocidad máxima puede ayudar a entrenadores y preparadores físicos a mejorar la planificación del entrenamiento de esta demanda competitiva. La especificidad del entrenamiento por grupos posicionales puede mejorar el entrenamiento individualizado del deportista, ya que las jugadoras de la línea de $3 / 4$ desarrollan unas mayores demandas con respecto a VMS, VMS en competición, umbral de sprint, número de sprint, distancia recorrida a sprint y sprints repetidos que las delanteras. Este hallazgo destaca la necesidad de programar ejercicios de entrenamiento directamente relacionado con las exigencias de cada grupo posicional.

Los datos muestran una pérdida de rendimiento en el segundo periodo, sugiriendo la necesidad de implantar programas de entrenamiento que potencien la capacidad de realizar acciones repetidas de alta intensidad y la VMS, adaptándose al nivel de exigencia de la competición para la mejora del rendimiento deportivo en el rugby 7 femenino.

El estudio además se ha realizado en competición, lo cual es un valor añadido, si bien, existe la limitación de no poder controlar las condiciones de atmosféricas de competición, las cuales podrían incidir sobre la velocidad.

\section{Referencias}

Agar-Newman, D. J., Goodale, T. L., \& Klimstra, M. D. (2017).
Anthropometric and physical qualities of international level female rugby sevens athletes based on playing position. Journal of Strength and Conditioning Research, 31(5), 1346-1352. https://doi.org/10.1519/JSC.0000000000001167

Al Haddad, H., Simpson, B. M., Buchheit, M., Di Salvo, V., \& Mendez-Villanueva, A. (2015). Peak match speed and maximal sprinting speed in young soccer players: Effect of age and playing position. International Journal of Sports Physiology and Performance, 10(7), 888-896. https://doi.org/10.1123/ ijspp.2014-0539

Austin, D., Gabbett, T., \& Jenkins, D. (2011). Repeated highintensity exercise in professional rugby union. Journal of Sports Sciences, 29(10), 1105-1112. https://doi.org/10.1080/ 02640414.2011 .582508

Barkell, J. F., O’Connor, D., \& Cotton, W. G. (2017). Situational coupling at the ruck and its effects on phase momentum and success in international men's and women's rugby sevens. Journal of Human Sport and Exercise, 12(2), 294-306. https:/ /doi.org/10.14198/jhse.2017.122.06

Blair, M. R., Body, S. F., \& Croft, H. G. (2017). Relationship between physical metrics and game success with elite rugby sevens players. International Journal of Performance Analysis in Sport, 17(4), 418-428. https://doi.org/10.1080/ 24748668.2017.1348060

Buchheit, M., Mendez-villanueva, A., Simpson, B. M., \& Bourdon, P. C. (2010). Repeated-sprint sequences during youth soccer matches. International Journal of Sports Medicine, 31(10), 709-716. https://doi.org/10.1055/s-0030-1261897

Clarke, A. C., Anson, J. M., \& Pyne, D. B. (2017). Game movement demands and physical profiles of junior, senior and elite male and female rugby sevens players. Journal of Sports Sciences, 35(8), 727-733. https://doi.org/10.1080/ 02640414.2016 .1186281

Cohen, J. (1988). Statistical power analysis for the behavioral sciences 2nd edn. Erlbaum Associates,.

den Hollander, S., Brown, J., Lambert, M., Treu, P., \& Hendricks, S. (2016). Skills associated with line breaks in elite rugby union. Journal of Sports Science \& Medicine, 15(3), 501-508. http:// www.ncbi.nlm.nih.gov/pubmed/27803629

Duthie, G. M., Pyne, D. B., Marsh, D. J., \& Hooper, S. L. (2006). Sprint patterns in rugby union players during competition. Journal of Strength and Conditioning Research, 20(1), 208214. https://doi.org/10.1519/R-16784.1

Duthie, G. M., Pyne, D., \& Hooper, S. (2005). Time motion analysis of 2001 and 2002 super 12 rugby. Journal of Sports Sciences, 23(5), 523-530. https://doi.org/10.1080/ 02640410410001730188

Gabbett, T. J. (2015). Use of relative speed zones increases the high-speed running performed in team sport match play. Journal of Strength and Conditioning Research, 29(12), 3353-3359. https://doi.org/10.1519/JSC.0000000000001016

Goodale, T. L., Gabbett, T. J., Stellingwerff, T., Tsai, M.-C., \& Sheppard, J. M. (2016). Relationship between physical qualities and minutes played in international women's rugby sevens. International Journal of Sports Physiology and Performance, 11(4), 489-494. https://doi.org/10.1123/ijspp.20140509

Goodale, T. L., Gabbett, T. J., Tsai, M.-C., Stellingwerff, T., \& Sheppard, J. (2017). The effect of contextual factors on physiological and activity profiles in international women's rugby sevens. International Journal of Sports Physiology \& Performance, 12(3), 370-376.

Higham, D. G., Pyne, D. B., Anson, J. M., Hopkins, W. G., \& Eddy, A. (2016). Comparison of activity profiles and physiological demands between international rugby sevens 
matches and training. Journal of Strength and Conditioning Research, 30(5), 1287-1294. https://doi.org/10.1097/ JSC.0000000000000212

Hodun, M., Clarke, R., De Ste Croix, M. B. A., \& Hughes, J. D. (2016). Global positioning system analysis of running performance in female field sports: a review of the literature. Strength and Conditioning Journal, 38(2), 49-56. https://doi.org/ 10.1519/SSC.0000000000000200

Jennings, D., Cormack, S., Coutts, A. J., Boyd, L. J., \& Aughey, R. J. (2010). Variability of GPS units for measuring distance in team sport movements. International Journal of Sports Physiology and Performance, 5(4), 565-569. http:// www.ncbi.nlm.nih.gov/pubmed/21266740

Jiménez-Reyes, P., Cross, M., Ross, A., Samozino, P., Brughelli, M., Gill, N., \& Morin, J.-B. (2019). Changes in mechanical properties of sprinting during repeated sprint in elite rugby sevens athletes. European Journal of Sport Science, 19(5), 585-594. https://doi.org/10.1080/17461391.2018.1542032

Lacome, M., Peeters, A., Mathieu, B., Bruno, M., Christopher, C., \& Piscione, J. (2019). Can we use GPS for assessing sprinting performance in rugby sevens? A concurrent validity and between-device reliability study. Biology of Sport, 36(1), 25 29.

Leal-Cussaria, J. A., Ortega-Gálvez, M. J., Porras-Alvarez, J., \& Galvez-Gonzalez, J. (2019). Demandas físicas y fisiológicas en el Hockey hierba femenino: diferencias entre los tiempos de juego. Retos, 35, 273-277.

Malone, S., Earls, M., Shovlin, A., Eddy, A., \& Winkelman, N. (2018). Match-play running performance and exercise intensity in elite international women's rugby sevens. Journal Of Strength And Conditioning Research, In press. https://doi.org/10.1519/ JSC.0000000000002547

Meir, R. (2012). Training for and competing in sevens rugby: practical considerations from experience in the International Rugby Board World Series. Strength and Conditioning Journal, 34(4), 76-86. https://doi.org/10.1519/SSC.0b013e31825105ed

Murray, N. B., Gabbett, T. J., \& Townshend, A. D. (2018). The use of relative speed zones in australian football: Are we really measuring what we think we are? International Journal of Sports Physiology and Performance, 13(4), 442-451. https://doi.org/ 10.1123/ijspp.2017-0148

Núñez-Sánchez, F. J., Toscano-Bendala, F. J., Campos-Vázquez, M. A., \& Suarez-Arrones, L. J. (2017). Individualized speed threshold to analyze the game running demands in soccer players using GPS technology. Retos, 32, 130-133. https:// recyt.fecyt.es/index.php/retos/article/view/52871

Núñez, F. J., Toscano-Bendala, F. J., Suarez-Arrones, L., MartínezCabrera, F. I., \& De Hoyo, M. (2018). Individualized speed thresholds to analyze acceleration demands in soccer players using GPS. Retos; Núm. 35 (2019), 32, 130-133. https:// recyt.fecyt.es/index.php/retos/article/view/60402

Ortega-Gálvez, M. J., Cabezas-Ramos, D., \& Gálvez-González, J. (2019). Cuantificación de las demandas de carrera en hockey hierba femenino mediante el uso de umbrales individualizados. RICYDE. Revista Internacional de Ciencias Del Deporte, 15(56), 123-136. https://doi.org/10.5232/ricyde2019.05601

Portillo, J., González-Ravé, J. M., Juárez, D., García, J. M., SuárezArrones, L., \& Newton, R. U. (2014). Comparison of running characteristics and heart rate response of international and national female rugby sevens players during competitive matches. Journal of Strength and Conditioning Research, 28(8), 2281-2289. https://doi.org/10.1519/JSC.0000000000000393

Reardon, C., Tobin, D. P., \& Delahunt, E. (2015). Application of individualized speed thresholds to interpret position specific running demands in elite professional rugby union: A GPS study. PLoS ONE, 10(7), 1-12. https://doi.org/10.1371/ journal.pone. 0133410

Ross, A., Gill, N., \& Cronin, J. (2014). Match analysis and player characteristics in rugby sevens. Sports Medicine (Auckland, N.Z.), 44(3), 357-367. https://doi.org/10.1007/s40279-0130123-0

Ross, A., Gill, N., \& Cronin, J. (2015). The match demands of international rugby sevens. Journal of Sports Sciences, 33(10), 1035-1041. https://doi.org/10.1080/02640414.2014.979858

Schuster, J., Howells, D., Robineau, J., Couderc, A., Natera, A., Lumley, N., Gabbett, T. J., \& Winkelman, N. (2018). Physicalpreparation recommendations for elite rugby sevens performance. International Journal of Sports Physiology and Performance, 13(3), 255-268. https://doi.org/10.1123/ijspp.20160728

Scott, M. T. U., Scott, T. J., \& Kelly, V. G. (2016). The validity and reliability of global positioning systems in team sport: a brief review. Journal of Strength and Conditioning Research, 30(5), 1470-1490. https://doi.org/10.1519/JSC.0000000000001221

Smart, D., Hopkins, W. G., Quarrie, K. L., \& Gill, N. (2014). The relationship between physical fitness and game behaviours in rugby union players. European Journal of Sport Science, 14(sup1), S8-S17. https://doi.org/10.1080/ 17461391.2011.635812

Suarez-Arrones, L., Arenas, C., López, G, Requena, B., Terrill, O., \& Mendez-Villanueva, A. (2014). Positional differences in match running performance and physical collisions in men rugby sevens. International Journal of Sports Physiology and Performance, 9(2), 316-323. https://doi.org/10.1123/ ijspp.2013-0069

Suarez-Arrones, L., Nuñez, F. J., Portillo, J., \& Mendez-Villanueva, A. (2012). Match running performance and exercise intensity in elite female rugby sevens. Journal of Strength and Conditioning Research, 26(7), 1858-1862. https://doi.org/ 10.1519/JSC.0b013e318238ea3e

Suarez-Arrones, L., Núñez, J., De Villareal, E. S., Gálvez, J., SuarezSanchez, G., \& Munguía-Izquierdo, D. (2016). Repeated-highintensity-running activity and internal training load of elite rugby sevens players during international matches: a comparison between halves. International Journal of Sports Physiology and Performance, 11(4), 495-499. https://doi.org/10.1123/ ijspp.2014-0523

Suarez-Arrones, L., Portillo, J., Pareja-Blanco, F., De Villareal, E. S., Sánchez-Medina, L., Munguía-Izquierdo, D. (2014). Match-play activity profile in elite women's rugby union players. Journal of Strength and Conditioning Research, 28(2), 452458. https://doi.org/10.1519/JSC.0b013e3182999e2b

Sweeting, A. J., Cormack, S. J., Morgan, S., \& Aughey, R. J. (2017). When is a sprint a sprint? A review of the analysis of teamsport athlete activity profile. Frontiers in Physiology, 8, 432. https://doi.org/10.3389/fphys.2017.00432

Vescovi, J., \& Goodale, T. (2015). Physical demands of women's rugby sevens matches: female athletes in motion (FAiM) study. International Journal of Sports Medicine, 36(11), 887-892. https://doi.org/10.1055/s-0035-1548940

Wheeler, K. W., \& Sayers, M. G. L. (2011). Rugby union contact skills alter evasive agility performance during attacking ball carries. International Journal of Sports Science \& Coaching, 6(3), 419-432. https://doi.org/10.1260/1747-9541.6.3.419

Zabaloy, S., Giraldez, J., Gazzo, F., Villaseca-vicuña, R., \& GalvezGonzalez, J. (2020). In-season assesment of sprint speed and sprint momentum in rugby players according to the age and playing position. Journal of Human Kinetics, In press. 